\title{
MicroRNA-379-5p suppresses renal fibrosis by regulating the LIN28/let-7 axis in diabetic nephropathy
}

\author{
NAN LI* , LI-JUAN WANG* ${ }^{*}$ WEI-LONG XU, SU LIU and JIANG-YI YU \\ Department of Endocrinology, Affiliated Hospital of Nanjing University of Chinese Medicine, \\ Nanjing, Jiangsu 210029, P.R. China
}

Received December 22, 2018; Accepted June 24, 2019

DOI: $10.3892 /$ ijmm.2019.4325

\begin{abstract}
MicroRNAs (miRNAs or miRs) play an important role in pathological processes in diabetic nephropathy (DN). This study aimed to explore whether miR-379-5p is associated with renal fibrosis in $\mathrm{DN}$ and to elucidate the underlying mechanisms. In vitro experiments indicated that miR-379-5p expression was downregulated by high glucose (HG) treatment in mouse mesangial cells (MMCs). Transfection with miR-379-5p mimics suppressed the proliferation and the accumulation of extracellular matrix (ECM) components, which were promoted by HG treatment. LIN28B was proven to be a direct target of miR-379-5p by luciferase report assay. In addition, the loss of expression of LIN28B, as well as the decrease in cell proliferation and in the accumulation of ECM components, which were induced by the knockdown of LIN28B, were attenuated in the MMCs following transfection with miR-379-5p inhibitors. Furthermore, type 2 diabetic $\mathrm{db} / \mathrm{db}$ mice were used to examine the efficiency of miR-379-5p agomir in the alleviation of renal fibrosis. Consistent with the results of the in vitro experiments, miR-379-5p agomir suppressed mesangial cell proliferation and the accumulation of ECM components by regulating the LIN28B/let-7 pathway. Taken together, the findings of this study suggest that miR-379-5p is highly involved in renal fibrosis in DN, and that it may be a potential effective therapeutic target for DN.
\end{abstract}

\section{Introduction}

As a main complication of diabetes mellitus, diabetic nephropathy (DN) is a leading cause of end-stage renal disease, accounting for $>35 \%$ of all new cases requiring

Correspondence to: Dr Jiang-Yi Yu, Department of Endocrinology, Affiliated Hospital of Nanjing University of Chinese Medicine, 155 Hanzhong Road, Nanjing, Jiangsu 210029, P.R. China E-mail: yujiangyi9506@126.com

*Contributed equally

Keywords: miR-379-5p, mesangial cell, diabetic nephropathy, LIN28B dialysis therapy globally (1). Clinically, DN is characterized by the development of proteinuria followed by decreased glomerular filtration (2). Pathologically, DN is characterized by the thickening of the basement membrane, renal tubal epithelial-mesenchymal transition (EMT), the accumulation of extracellular matrix (ECM) components, glomerular sclerosis and tubulointerstitial fibrosis (3). However, there is limited acknowledge available on the pathological mechanisms involved in DN, and thus there is also a lack of effective treatments for DN. Therefore, it is of utmost importance to study its precise molecular mechanisms and to develop novel effective therapeutic strategies for DN.

MicroRNAs (miRNAs or miRs) are small non-coding single-stranded RNAs which have been confirmed to play critical roles in cell proliferation, apoptosis and differentiation, contributing to the pathogenesis of a number of human deceases, such as cancer and diabetes $(4,5)$. Researches have indicated that miRNAs are highly involved in DN, which suggests the great potential of miRNAs in DN detection, as well as therapy $(6,7)$. It has been found that urinary miRNAs related to histopathological lesions and kidney damage can be applied to the sensitive, specific and non-invasive detection of DN (8). Furthermore, miRNAs have been successfully applied to DN therapy. The addition of the agomir of the downregulated miRNAs (i.e., miR-455-3p) or antagomir of the commonly upregulated miRNAs (i.e., miR-192 and miR-377) in DN has been shown to induce a decrease in albuminuria and mesangial matrix accumulation in animal models (9-11).

miR-379-5p has been reported to be downregulated in spontaneous type 2 diabetic KKay mice (12). In this study, we investigated the role of miR-379-5p in DN both in vitro and in vivo. Glomerular hypertrophy, mainly caused by glomerular mesangial cell (MC) proliferation and the accumulation of ECM components, is considered to be one of the earliest pathological characteristics of DN. Of the three types of constitutive cells in the glomerulus, MCs are the main source of ECM proteins. Therefore, we isolated and cultured mouse glomerular MCs (MMCs) for experiments in vitro. LIN28B, the direct target of miR-379-5p, was identified by the database, TargetScan. LIN28B has been shown to be closely related to various types of cancer (13). It has been reported that in MMCs treated with transforming growth factor (TGF)- $\beta$, the LIN28/let-7 pathway can affect the accumulation of ECM 
components by targeting collagen type 1- $\alpha 2$ (Col1a2) and collagen type 4- $\alpha 1$ (Col4a1) (14). In this study, we attempted to investigate whether miR-379-5p regulates the LIN28/let-7 axis in $\mathrm{DN}$, in an aim to provide a promising approach for the clinical treatment of DN.

\section{Materials and methods}

Cell culture and transfection. MMCs were isolated from 8 -week-old male C57BL/6 mice $(n=6)$ as previously described (15). The mice were euthanized by an intraperitoneal injection of sodium pentobarbital $(100 \mathrm{mg} / \mathrm{kg})$. All experiments were approved by the Animal Care and Use Committee of the Nanjing University of Chinese Medicine and complied with the Declaration of the National Institutes of Health Guide for the Care and Use of Laboratory Animals. MMCs were obtained from the renal cortex of the 8-week-old male mice using sterile techniques. After washing with RPMI-1640 medium (Beyotime Biotechnology), the cortices were separated from the medullas, pooled, minced and the glomeruli were isolated. The glomeruli were then pre-treated with collagenase for 15 min and plated in RPMI-1640 medium supplemented with $10 \%$ fetal bovine serum (FBS) and $20 \mu \mathrm{g} / \mathrm{ml}$ insulin at $37^{\circ} \mathrm{C}$ with $5 \% \mathrm{CO}_{2}$. Cells at passages between 3 and 7 were used in all the experiments.

The MMCs were treated with normal glucose $(5 \mathrm{mmol} / \mathrm{l})$ plus mannitol $(25 \mathrm{mmol} / \mathrm{l})$ for the control group and high glucose $(30 \mathrm{mmol} / \mathrm{l})$ for the high glucose $(\mathrm{HG})$ group. miR-379-5p mimics, inhibitors, agomir and siRNA against LIN28B and the negative controls were purchased from RiboBio (Guangzhou). All of the transient transfections were conducted using Lipofectamine 2000 reagent (Invitrogen; Thermo Fisher Scientific). 293T cells were acquired from the Chinese Academy of Sciences Cell Bank and were cultured in Dulbecco's modified Eagle's medium (DMEM) containing 10\% FBS.

Luciferase reporter assay. The LIN28B transcript sequences were acquired from NCBI resources (gene ID, 380669). The 3' UTR of LIN28B with predicted target sites for miR-379-5p seed sequence was amplified and cloned into the PGL-3 vector (Promega) using KpnI/HindIII sites. The primers used were as follows: Sense, 5'-GGTACCGCCTTTGATTCAGAA ACGG-3' and antisense, 5'-AAGCTTCTATAAAACATG ACACCCGC-3'. The mutated 3' UTR of LIN28B was generated using the QuikChange II Site-Directed Mutagenesis kit (Stratagene). At $48 \mathrm{~h}$ following the co-transfection of miR-379-5p mimics (100 $\mathrm{nM})$ and the wild-type or mutated 3' UTR of LIN28B, the luciferase activity of the 293T cells was measured using the dual-luciferase assay kit (Promega) with Renilla luciferase as the internal control.

Reverse transcription-quantitative PCR (RT- $q P C R)$. Total RNA was extracted from the MMCs and renal tissues (see below) using TRIzol reagent (Invitrogen; Thermo Fisher Scientific). cDNA was synthesized using the PrimeScript ${ }^{\circledR}$ RT reagent kit (Takara). qPCR was performed using SYBR Premix ExTaq ${ }^{\mathrm{TM}}$ (Takara) on the platform of Applied Biosystems 7500. U6 was used as an endogenous normalization control. The total reaction system of $20 \mu$ l volume was as follows: $1 \mu \mathrm{l}$ cDNA, $10 \mu \mathrm{l}$ SYBR Premix EX Taq, $1 \mu \mathrm{l}$ each of the primers $(10 \mu \mathrm{M})$ and
$7 \mu 1 \mathrm{ddH}_{2} \mathrm{O}$. The PCR cycling conditions were as follows: $95^{\circ} \mathrm{C}$ for $5 \mathrm{~min}$, followed by 40 cycles of $95^{\circ} \mathrm{C}$ for $15 \mathrm{sec}$ and $60^{\circ} \mathrm{C}$ for $30 \mathrm{sec}$. The primers used were as follows: miR-379-5p forward, 5'-GCGCTGGTAGACTATGGAA-3' and reverse, 5'-GTGCAGGGTCCGAGGT-3'; U6 forward, 5'-CTCGCT TCGGCAGCACATATACT-3' and reverse, 5'-ACGCTTCAC GAATTTGCGTGTC-3'; Lin28B forward, 5'-GTCAATACG GGTAACAGGAC-3' and reverse, 5'-TTCTTTGGCTGA GGAGGTAG-3'; Let-7b forward, 5'-TGAGGTAGTAGG TTGTGT-3' and reverse, 5'-GTCGTATCCAGTGCAGGG TCCGAGGT-3'; Col-1 forward, 5'-CCACCCCAGCCGCAA AGAGTC-3' and reverse, 5'-GTCATCGCACACAGCCGT GC-3'; Col-4 forward, 5'-ATCTCTGCCAGGACCAAGTG-3' and reverse, 5'-CGGGCTGACATTCCACAAT-3'; GAPDH forward, 5'-GAAATCCCATCACCATCTTCCAGG-3' and reverse, 5'-GAGCCCCAGCCTTCTCCATG-3'. Data analyses were performed using the comparative $\mathrm{Cq}(\Delta \Delta \mathrm{Cq})$ method for calculating relative gene expression (16).

Western blot analysis. Renal tissue (see below) and MMCs were lysed in radioimmunoprecipitation buffer. Approximately $80 \mu \mathrm{g}$ of total proteins were separated by $12 \%$ or $10 \%$ SDS-PAGE gel and then transferred onto PVDF membranes (Millipore Corp.) by electroblotting. After being blocked for $60 \mathrm{~min}$ with $5 \%$ non-fat milk, the membranes were incubated at $4{ }^{\circ} \mathrm{C}$ overnight with primary antibodies against Col4 (ab6586; 1:1,000), fibronectin (FN; ab2413; 1:1,000), p21 (ab109199; $1: 1,000)$, cyclin D1 (ab134175; 1:1,000) (all from Abcam) and LIN28B (\#5422; 1:1,000; Cell Signaling Technology). GAPDH (ab9485; 1:2,000; Abcam) was served as the loading control. The membranes were then incubated with the secondary antibodies anti-rabbit IgG, HRP-linked antibody $(1: 1,000, \# 7074$, Cell Signaling Technology) for $1 \mathrm{~h}$ at room temperature. Subsequently, they were visualized with ECL detection reagent (Millipore), and the gray values of the bands were calculated automatically (ImageJ software, version 4.3).

Cell proliferation. Cell viability was assayed using the Cell Counting kit-8 (Dojindo) following the manufacturer's instructions. The cells were seeded in 96-well plates at a density of $2 \times 10^{3}$ cells per well. CCK- 8 solution $(10 \mu \mathrm{l})$ was added to each well for $1 \mathrm{~h}$ following $48 \mathrm{~h}$ of incubation, and OD450 was determined using a microplate reader (ELx808, BioTek). Moreover, 5-ethynyl-2'-deoxyuridine (EdU) assay (Beyotime Biotechnology) was performed to detect the proliferation of the MMCs. Following incubation with $10 \mu \mathrm{M} \mathrm{EdU} \mathrm{for} 2 \mathrm{~h}$ at $37^{\circ} \mathrm{C}$, the cells were fixed in $4 \%$ paraformaldehyde. Subsequently, Hoechst 33342 (Beyotime Biotechnology) was used to stain the nuclei at room temperature for $30 \mathrm{~min}$. Finally, the cells were visualized under a fluorescence microscopy (Leica).

Cell cycle analysis. For cell cycle analysis, the MMCs were harvested, suspended in $500 \mu 1$ of phosphate-buffered saline (PBS), fixed with 70\% ethanol and subjected to PI/RNase (Beyotime Biotechnology) staining for $30 \mathrm{~min}$ at $4^{\circ} \mathrm{C}$. The MMCs were then analyzed by flow cytometry (FACSCanto II, BD Biosciences).

Animal experiments. A total of $18 \mathrm{C} 57 \mathrm{BL} / \mathrm{KsJ}$ type 2 diabetic $\mathrm{db} / \mathrm{db}$ male mice (aged 4 weeks, weighing 18-22 g) and a total 
of 6 heterozygote $\mathrm{db} / \mathrm{m}$ male mice (aged 4 weeks, weighing 18-22 g) used in this study were purchased from the Model Animal Research Center of Nanjing University. The mice were housed in a clean environment with 50-60\% humidity, 20-22 ${ }^{\circ} \mathrm{C}$ and a $12-\mathrm{h}$ dark/light cycle, and fed a standard diet during a 1 -week adaptation period. When the mice were 8 weeks old, they were randomly divided into the control group (untreated $\mathrm{db} / \mathrm{m}$ mice, $\mathrm{n}=6$ ); the $\mathrm{DN}$ group (untreated $\mathrm{db} / \mathrm{db}$ mice, $\mathrm{n}=6$ ); an agomir NC (agomir NC-treated $\mathrm{db} / \mathrm{db}$, $\mathrm{n}=6$ ) group and an agomir-379-5p (agomir-379-5p-treated $\mathrm{db} / \mathrm{db}, \mathrm{n}=6$ ) group. Every 2 days, the $\mathrm{db} / \mathrm{db}$ mice were injected with miR-379-5p agomir or agomir NC $(10 \mathrm{mg} / \mathrm{kg})$ (GenePharma) via the tail vein. Four weeks later, the mice were sacrificed by an intraperitoneal injection of sodium pentobarbital $(100 \mathrm{mg} / \mathrm{kg})$ and the kidneys were harvested for analysis. During the experiments, animal health and behavior were monitored every 2 days. All experiments were approved by the Animal Care and Use Committee of the Nanjing University of Chinese Medicine and complied with the Declaration of the National Institutes of Health Guide for the Care and Use of Laboratory Animals.

Measurement of urine protein levels. Metabolic cages were used to collect urine samples in a 24-h period, and urinary protein levels were evaluated using the mouse albumin ELISA quantitation set (Bethyl Laboratories).

Histology and immunostaining assays. Fresh renal tissues were fixed with $4 \%$ paraformaldehyde and embedded in paraffin. Paraffin-embedded specimens were sectioned (4 $\mu \mathrm{m}$ thicknesses) and stained with PAS, Masson's trichrome staining (Beyotime Biotechnology) at room temperature for $15 \mathrm{~min}$ and immunohistochemistry. The antibodies included LIN28B (ab71415; 1:50), Ki-67 (ab15580; 1:500) and normal rabbit IgG (ab6728, 1:1,000) (all from Abcam) were incubated for $2 \mathrm{~h}$ at $37^{\circ} \mathrm{C}$.

In situ hybridization(ISH). To detect the presence of miR-379-5p in the renal tissues, in situ hybridization was performed. The frozen renal tissues sections of mice from control group (untreated $\mathrm{db} / \mathrm{m}$ mice), DN group (untreated $\mathrm{db} / \mathrm{db}$ mice) and agomir-379-5p (agomir-379-5p-treated db/db mice) group were fixed in paraformaldehyde followed by rinsing in PBS. A digoxin-labeled oligonucleotide probe (5'-TGGTAGACTATG GAACGTAGG-3') $(0.5 \mu \mathrm{g} / \mathrm{ml})$ was synthesized by Dingguo BioTechnology Co. Ltd. In situ hybridization was performed with the ISH kit according to the protocol (Dingguo BioTech). The slides were treated with 3,3'-diaminobenzidine (DAB) for $5 \mathrm{~min}$, and then counterstained with hematoxylin for 1-2 min at room temperature. All images were acquired by using a light microscope (Olympus) at the magnification of $\mathrm{x} 400$.

Bioinformatics analysis. TargetScan (http://www.targetscan. org/) was used to predict the putative target genes for miR-379-5p.

Statistical analysis. GraphPad Prism (version 5.0; GraphPad Software, Inc.) was used to conduct all statistical analyses. Measurement data are presented as the means \pm standard deviation. One-way ANOVA analysis followed by a Tukey's post hoc test were used to compare the difference between multiple groups. Values of $\mathrm{P}<0.05$ and $\mathrm{P}<0.01$ were considered to indicate statistically significant and highly statistically significant differences, respectively.

\section{Results}

miR-379-5p is downregulated by high glucose treatment in $M M C s$. To explore the potential role of miR-379-5p in DN, we analyzed the expression of miR-379-5p in MMCs stimulated with HG. As shown in Fig. 1A and B, miR-379-5p expression was significantly decreased in the cells treated with high glucose in a dose- and time-dependent manner, and its expression was approximately one third of that of the control following stimulation of the cells with $30 \mathrm{mM}$ glucose for $48 \mathrm{~h}$.

miR-379-5p suppresses the proliferation and accumulation of ECM components in MMCs. Mesangial cell proliferation and the accumulation of ECM components are important pathological features of DN (17). In this study, to explore the effects of miR-379-5p on cell proliferation and the accumulation of ECM components, the MMCs were transfected with miR-379-5p mimics. The results revealed that miR-379-5p expression was markedly increased in the MMCs transfected with miR-379-5p mimics (Fig. 1C). The results of CCK-8 assay also revealed that $\mathrm{HG}$ stimulation markedly promoted the viability of the MMCs. However, the promoting effects of HG stimulation on cell viability were reversed following transfection of the MMCs with miR-379-5p mimics (Fig. 2A). The results of EdU assays yielded similar conclusions, in that transfection with miR-379-5p mimics attenuated the effects of HG stimulation on cell proliferation (Fig. 2B). Furthermore, cell cycle analysis indicated that there was a decrease in the percentage of cells in the $G_{0} / G_{1}$ phase, as well as an increase in the percentage of cells in the $\mathrm{S}$ phase in the HG group compared with the control group. MMCs transfected with miR-379-5p mimics exhibited an increase in the percentage of cells in the $G_{0} / G_{1}$ phase and a decrease in the percentage of cells in the $\mathrm{S}$ phase compared with the HG group (Fig. 2C). This suggested that the overexpression of miR-379-5p prevented MMCs cell cycle progression.

p21, a cyclin-dependent kinase inhibitor, almost inhibits all cyclin/CDK complexes. The expression level of p21 and cyclin D1 can reflect the proliferation of MMCs (18). In this study, the results of western blot analysis revealed that transfection with miR-379-5p mimics attenuated the decrease and increase in the expression level of p21 and cyclin D1, respectively induced by HG stimulation (Fig. 2D). The cell cycle analysis and the related protein experimental data suggested that the overexpression of miR-379-5p suppressed the proliferation of MMCs following HG stimulation. Tubulointerstitial fibrosis is always involved with the increased deposition of ECM proteins, such as collagens and FN $(19,20)$. In this study, we detected Col4 and FN expression by western blot analysis. As shown in Fig. 2E, Col4 and FN expression levels were markedly promoted in the HG group and the mimics-NC group compared to the control group, and decreased in the mimics group compared to the mimics-NC group. Taken together, these results indicated that miR-379-5p suppressed the proliferation and accumulation of ECM components in MMCs. 

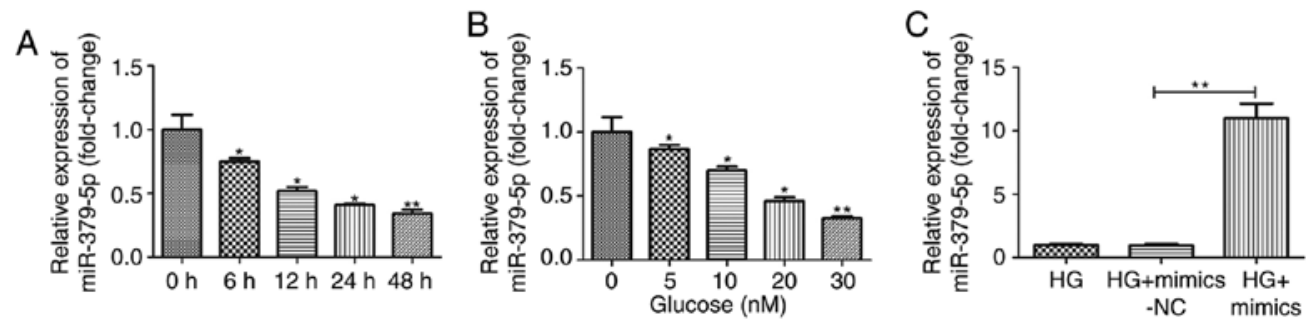

Figure 1. miR-379-5p expression is downregulated by HG treatment in MMCs. Expression of miR-379-5p was detected by RT-qPCR in MMCs treated with (A) $30 \mathrm{mM}$ glucose for $0,6,12,24$ and $48 \mathrm{~h}$ or (B) with various concentration of glucose $(0,5,10,20$ and $30 \mathrm{mM})$ for $24 \mathrm{~h}$. (C) Following transfection with mimics-NC or miR-379-5p mimics, the cells were treated with HG $(30 \mathrm{mmol} / \mathrm{l})$ for $24 \mathrm{~h}$, and miR-379-5p expression was then detected by RT-qPCR. Data are expressed as the mean \pm SEM. ${ }^{*} \mathrm{P}<0.05$ and ${ }^{* * *} \mathrm{P}<0.01$ compared with the control group or HG group. HG, high glucose; MMCs, mouse mesangial cells.
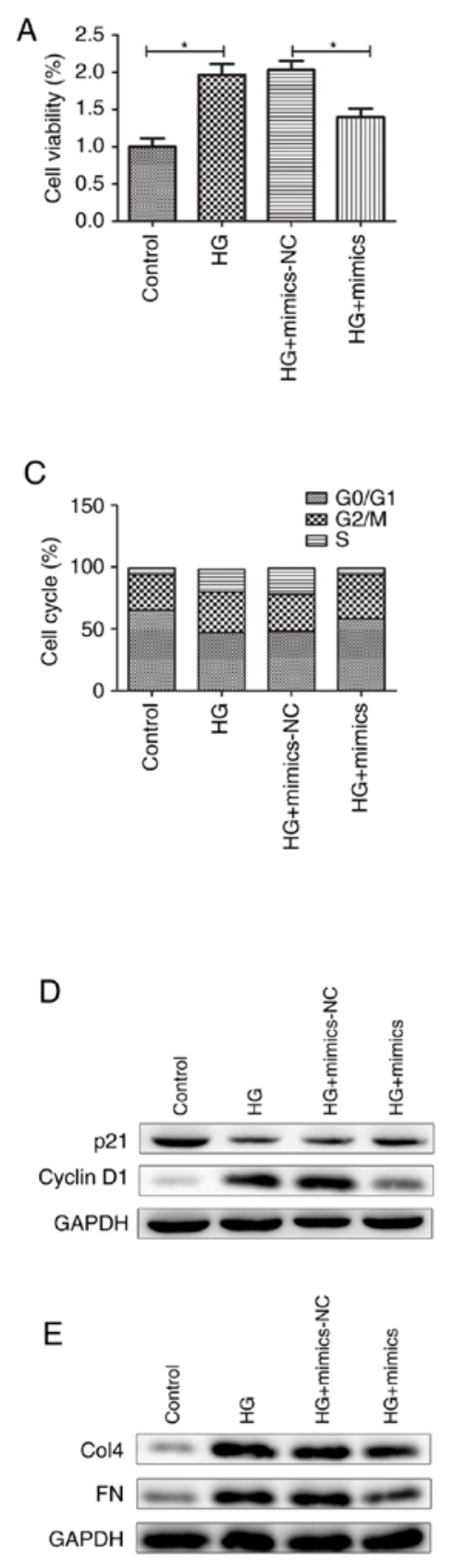
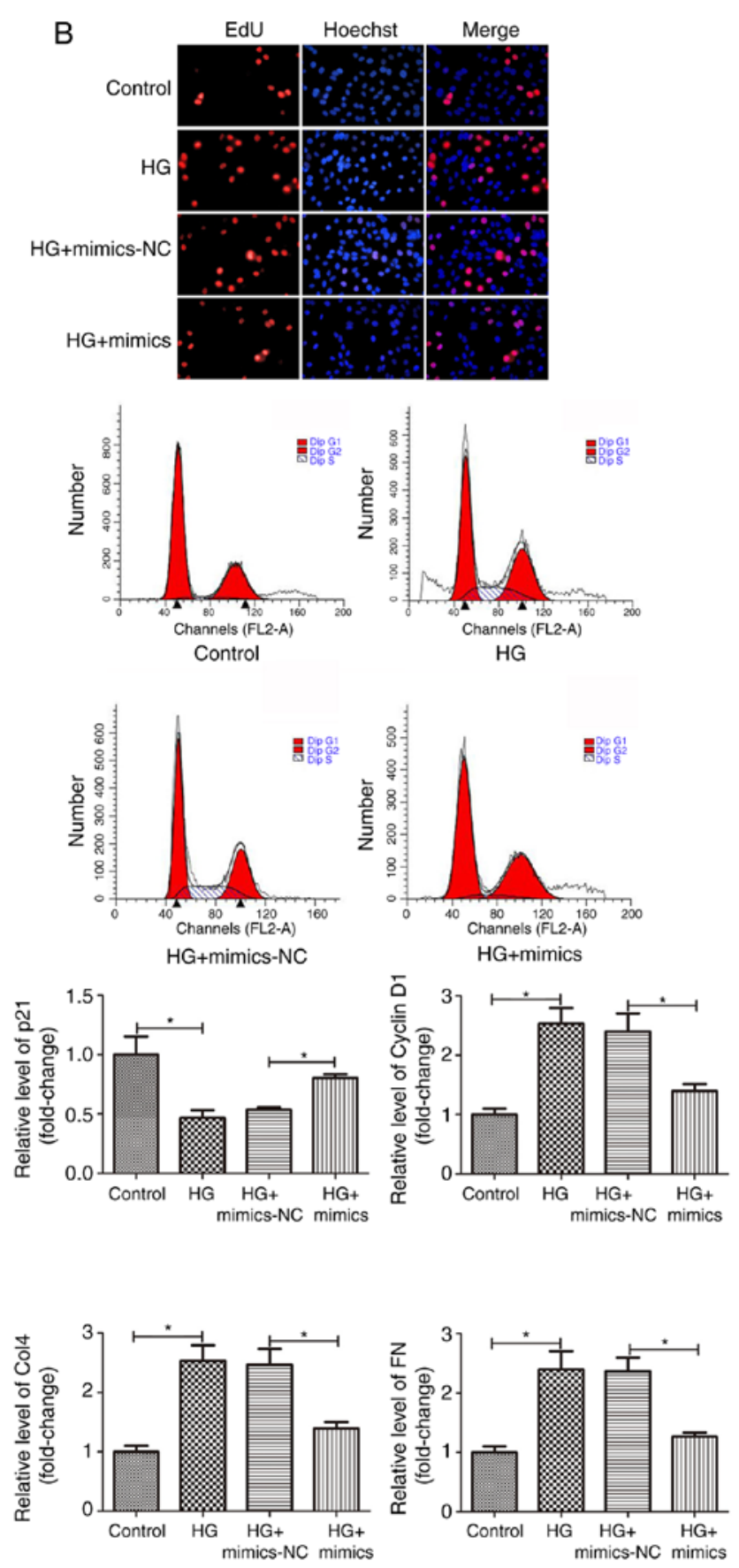

Figure 2. miR-379-5p suppresses the proliferation and accumulation of ECM components in MMCs. (A) CCK-8 assays; (B) EdU assays; (C) Cell cycle analysis; (D) western blot analysis for $\mathrm{p} 21$ and cyclin D1; (E) western blot analysis for Col-4 and FN in the control group; HG group; HG + mimics-NC group and HG + miR-379-5p mimics group. Data are expressed as the means \pm SEM. ${ }^{*} \mathrm{P}<0.05$ compared with the control group or HG group. HG, high glucose; MMCs, mouse mesangial cells; ECM, extracellular matrix. 
A LIN28B3'UtR $5^{\prime}$...UUACCCGGUUGCAAAGUCUACCU... hsa-miR-379-5p 3' GGaUgCaAgGuaucagaugGU
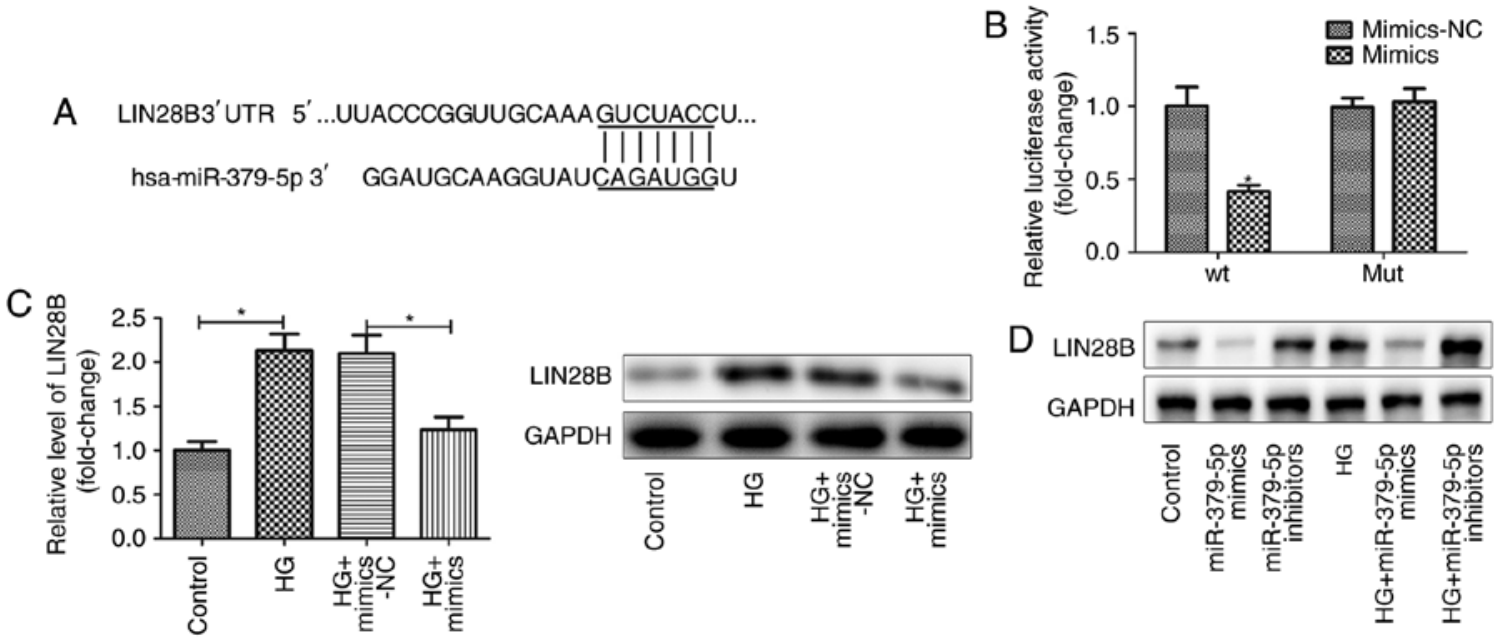

Figure 3. LIN28B is target of miR-379-5p. (A) Sequence alignment of the putative binding sites of miR-379-5p in the 3' UTR of LIN28B that was predicted by TargetScan software. (B) Luciferase reporter assays: Relative luc activity in miR-379-5p mimics or mimics NC transfected MMCs was measured following co-transfection with wild-type or mutated 3' UTR of LIN28B. (C) Western blot analysis for LIN28B in the control group; HG group; HG + mimics-NC group and HG + miR-379-5p mimics group. (D) The western blot analysis data revealed that miR-379-5p inactivated LIN28B expression in a HG-independent manner. Data are expressed as the means \pm SEM. ${ }^{*} \mathrm{P}<0.05$ compared to luc activity in MMCs cotransfected with mimics NC and the wild-type 3 ' UTR of LIN28B or compared with the control group or HG group. HG, high glucose; MMCs, mouse mesangial cells.

LIN28B is a target of miR-379-5p. The TargetScan database was used to identify the targets of miR-379-5p. It was suggested that miR-379-5p had a conserved binding site in the 3' UTR of LIN28B (Fig. 3A). To verify the association between miR-379-5p and LIN28B, luciferase reporter assays were performed. The results revealed that the luciferase activity was significantly decreased in the MMCs co-transfected with miR-379-5p mimics and the wild-type 3' UTR reporter gene other than the mutated 3 ' UTR reporter gene, indicating that miR-379-5p directly binds to the 3' UTR of LIN28B (Fig. 3B).

LIN28B, a RNA-binding protein belongs to LIN28 family, mediates diverse biological functions (21). In this study, to examine the regulatory association between miR-379-5p and LIN28B, the expression levels of LIN28B and let-7b were detected in MMCs transfected with miR-379-5p mimics. As shown in Fig. 3C, the overexpression of miR-379-5p markedly suppressed the LIN28B expression level, which was induced by HG. The results of western blot analysis also revealed that miR-379-5p inactivated LIN28B expression in a HG-independent manner (Fig. 3D).

miR-379-5p suppresses the proliferation and accumulation of ECM components by regulating LIN28B expression. Previously, Kim et al demonstrated that LIN28B increased the expression of ECM proteins, such as Col1a2 and Col4a1, by decreasing let-7 levels (22). In this study, firstly, the transfection efficiency of miR-379-5p inhibitor was evaluated by RT-qPCR. The results revealed that the cells transfected with miR-379-5p inhibitor exhibited lower levels of miR-379-5p expression compared with the negative control group, indicating that miR-379-5p inhibitor was transfected into the cells successfully (Fig. 4A). To explore whether miR-379-5p suppresses the proliferation and accumulation of ECM components by regulating the LIN28/let-7 axis, a si-LIN28B vector was constructed and transfected into the MMCs (Fig. 4B and C). Transfection with si-LIN28B decreased LIN28B expression; however, miR-379-5p inhibitor partly reversed the decrease in LIN28B induced by si-LIN28B in the cells treated with HG (Fig. 4B and C). CCK-8 assay was performed in the HG group, si-NC group, inhibitors-NC group, si-LIN28B group and si-LIN28B + miR-379-5p inhibitors group to examine the effects of LIN28B on MMC viability. The results revealed that the knockdown of LIN28B suppressed MMC viability (Fig. 4D). Transfection with si-LIN28B decreased the mRNA expression levels of Coll and Col4, and increased the let-7 expression level, as shown by RT-qPCR (Fig. 4E). Western blot analysis for Col-1 and Col-4 revealed that si-LIN28B decreased the accumulation of the ECM components, Col-1 and Col-4, compared to si-NC group (Fig. 4F). However, MMCs co-transfected with miR-379-5p inhibitors and si-LIN28B exhibited a higher proliferation rate and a greater accumulation of ECM components compared to the corresponding si-LIN28B group (Fig. 4D-F). Taken together, these results suggested that miR-379-5p suppressed the proliferation and accumulation of ECM components by regulating the LIN28/let-7 axis.

miR-379-5p suppresses renal fibrosis in vivo. To examine the effects of miR-379-5p on renal fibrosis in DN, we used type 2 diabetic $\mathrm{db} / \mathrm{db}$ mice for research in vivo with $\mathrm{db} / \mathrm{m}$ mice as a normal control. miR-379-5p expression levels in the kidney tissues were significantly downregulated in the $\mathrm{db} / \mathrm{db}$ mice, as detected by RT-qPCR and in situ hybridization assays. miR-379-5p agomir injection markedly promoted miR-379-5p expression (Fig. 5A and B). Consequently, LIN28B expression was upregulated in the kidney tissues of the $\mathrm{db} / \mathrm{db}$ mice and downregulated after the injection of miR-379-5p agomir (Fig. 5C-E). Moreover, glomerular hypertrophy with an increased glomerular area was observed 

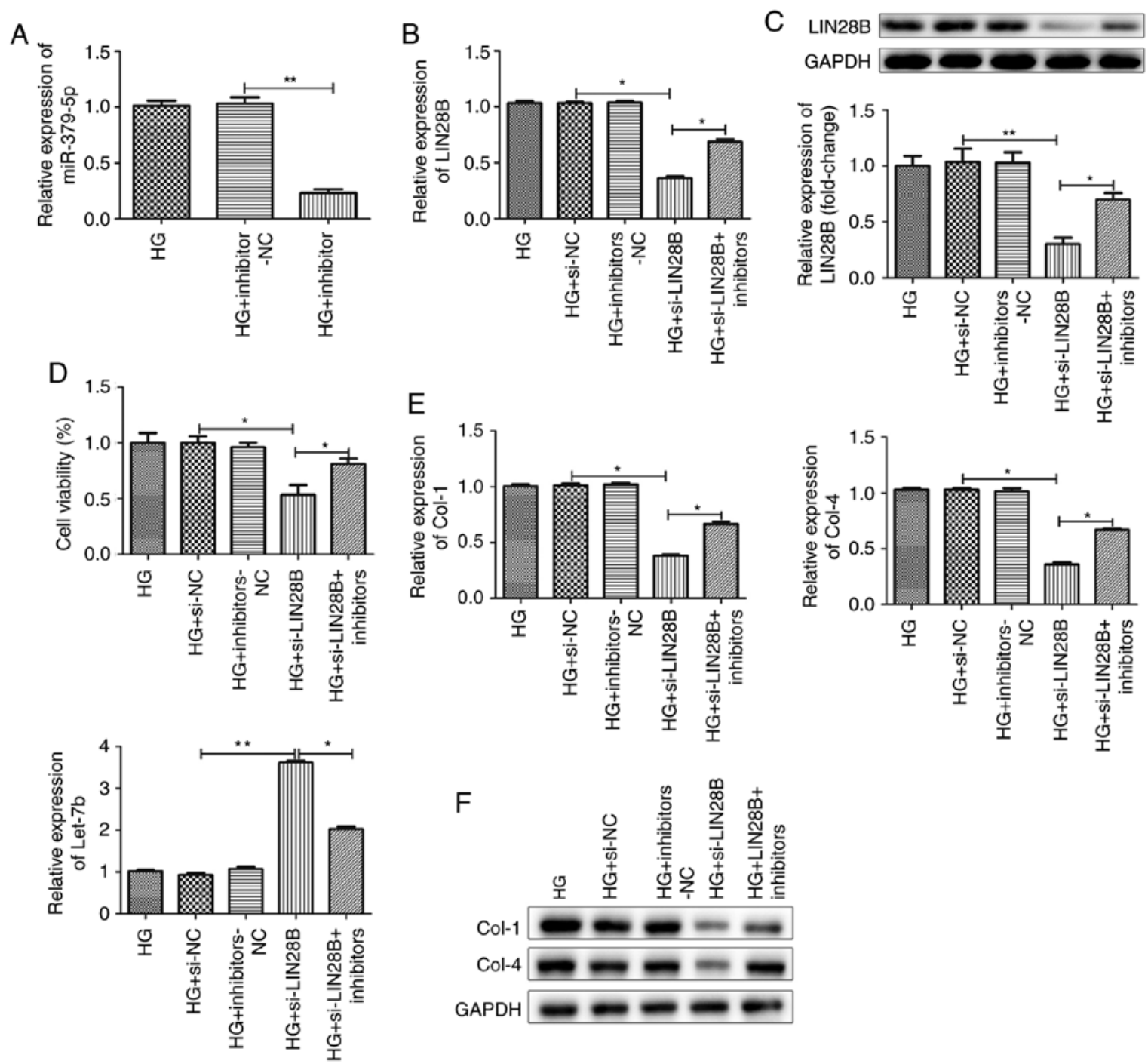

Figure 4. miR-379-5p suppresses the proliferation and accumulation of ECM components by regulating LIN28B. (A) Transfection efficiency of miR-379-5p was detected by RT-qPCR. (B and C) RT-qPCR and western blot analysis for LIN28B in MMCs transfected with si-NC or si-LIN28B. Data are expressed as the means \pm SEM. ${ }^{*} \mathrm{P}<0.05$ and ${ }^{* *} \mathrm{P}<0.01$ compared to si-NC group. (D) CCK-8 assays; (E) RT-qPCR assays for Col-1, Col-4 and Let-7b expression. (F) Western blot analysis for Col-1 and Col-4 in HG treated MMCs and HG treated MMCs transfected with si-NC; inhibitors-NC or si-LIN28B and MMCs co-transfected with miR-379-5p inhibitors and si-LIN28B. Data are expressed as the means \pm SEM. ${ }^{*} \mathrm{P}<0.05$ and ${ }^{* *} \mathrm{P}<0.01$ compared to si-NC group or si-LIN28B group. HG, high glucose; MMCs, mouse mesangial cells; ECM, extracellular matrix.

in the $\mathrm{db} / \mathrm{db}$ mice. However, miR-379-5p agomir attenuated glomerular hypertrophy. As is well-known, the earliest pathological characteristic of DN is glomerular hypertrophy, which is mainly caused by glomerular MC proliferation and ECM accumulation. In this study, we wished to determine the role of the miR-379-5p/LIN28/let-7 axis in glomerular MCs during DN. Physiological markers, such as urine protein and blood glucose were also measured. It was found that urine protein levels were significantly increased in the $\mathrm{db} / \mathrm{db}$ mice, and urine protein levels were decreased in the miR-379-5p agomir group (Fig. 5F).

In order to examine the effects of miR-379-5p on renal fibrosis in DN, some pathological markers, such as PAS and Masson's trichrome staining were used (Fig. 6A). The results indicated that miR-379-5p alleviated pathological changes, such as glomerular hypertrophy, mesangial amplification and renal fibrosis in the diabetic mice. Furthermore, mesangial cell proliferation detected by $\mathrm{Ki}-67$ staining is presented in
Fig. 6B, and ECM accumulation was measured by RT-qPCR and western blot analysis for let-7b, Col-4 and Col-1 expression (Fig. 6C and D). The results revealed that miR-379-5p agomir decreased mesangial cell proliferation and accumulation of ECM components that were increased in the $\mathrm{db} / \mathrm{db}$ mice, which was consistent with the data obtained from the in vitro assays.

\section{Discussion}

Recently, increasing evidence has indicated that the dysregulation of miRNAs, such as miR-214, miR-15a, miR-29a and miR-34a plays an important role in the pathogenesis of DN (23-31). In this study, we found that miR-379-5p was downregulated and acted as a suppressor of renal fibrosis by regulating LIN28B in diabetic nephropathy.

miR-379 has been found to participate in a number of biological processes. It acts as a tumor suppressor and is 


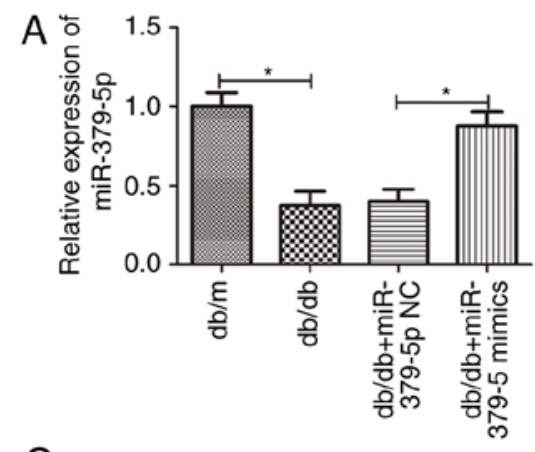

B
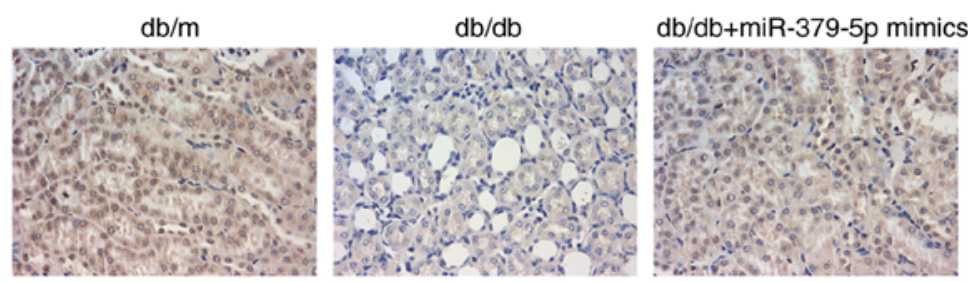

C

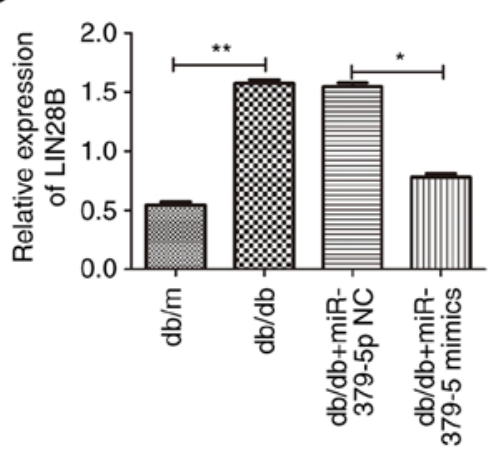

D
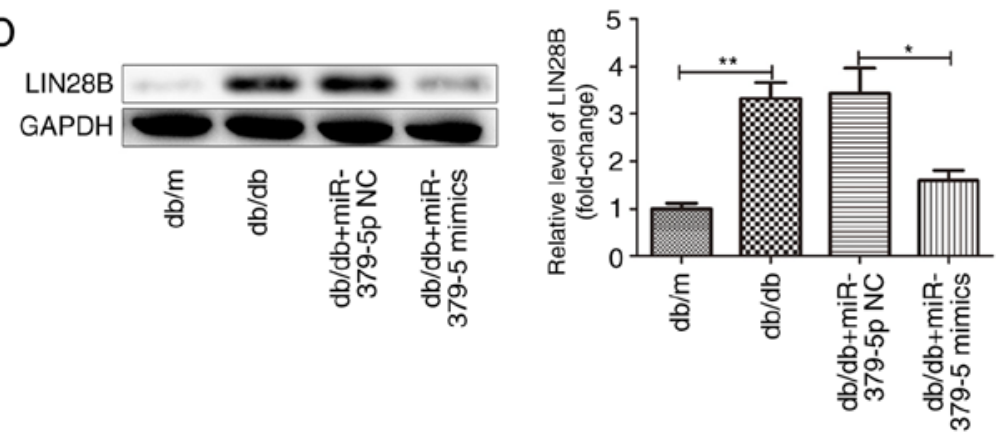

E

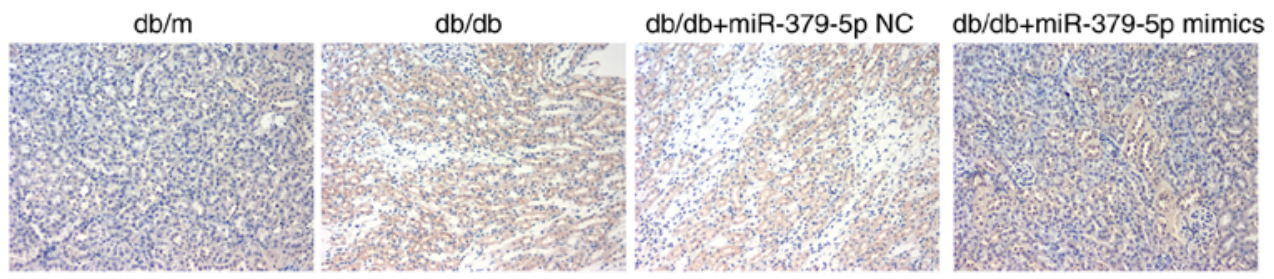

$\mathrm{F}$
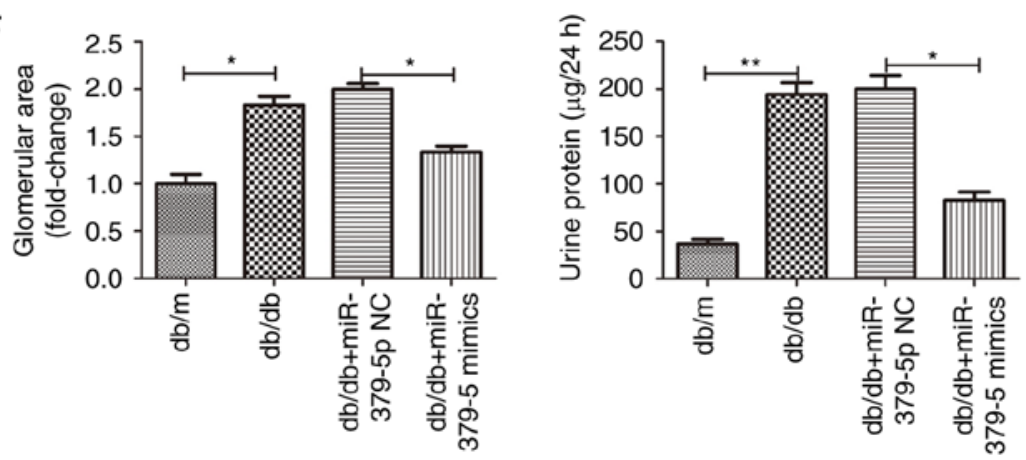

Figure 5. miR-379-5p suppresses renal fibrosis in vivo. (A) Expression levels of miR-379-5p were detected by RT-qPCR in the kidney tissues in db/m mice; $\mathrm{db} / \mathrm{db}$ mice and db/db mice with miR-379-5p injection by the tail vein. (B) expression of miR-379-5p was detected by in situ hybridization (magnification, x400). (C) RT-qPCR; (D) Western blot analysis; (E) immunohistochemical staining for LIN28B in the glomeruli (magnification, x200). (F) Urine protein and blood glucose levels. Data are expressed as the means \pm SEM. ${ }^{*} \mathrm{P}<0.05$ and ${ }^{* *} \mathrm{P}<0.01$ compared to db/db mice or to NC group.

downregulated in human cancers (32-34). In hepatocellular carcinoma and non-small-cell lung cancer, miR-379 is associated with chemosensitivity $(35,36)$. Moreover, miR-379 has been shown to be downregulated in patients with atherosclerotic coronary artery disease and acute myocardial infraction $(37,38)$.

LIN28B is an oncogene that has been reported to be overexpressed in numerous malignant tumors (39-42). LIN28A AND LIN28B selectively block the expression of let-7 miRNAs and promote tumorigenesis (43). On the other hand, the LIN28B/let-7 axis has been reported to be involved in controlling TGF- $\beta$-induced collagen accumulation in
DN (14). In addition, LIN28B has been reported to be associated with fibrosis lesions. For instance, LIN28B can participate in the progression of organ damage and fibrosis in metabolic diseases, such as diabetes (14). LIN28B can ameliorate liver fibrosis by inhibiting mesenchymal signaling pathways (44). The upregulation of LIN28B has been shown to contribute to idiopathic pulmonary fibrosis (45). It is well known that transforming growth factor- $\beta 1$ (TGF- $\beta 1$ ) expression is increased in glomerular MCs that lead to an increased accumulation of ECM components and hypertrophy during the progression of DN (46). TGF- $\beta 1$ can induce LIN28B expression which results in the suppression of let-7 and the 


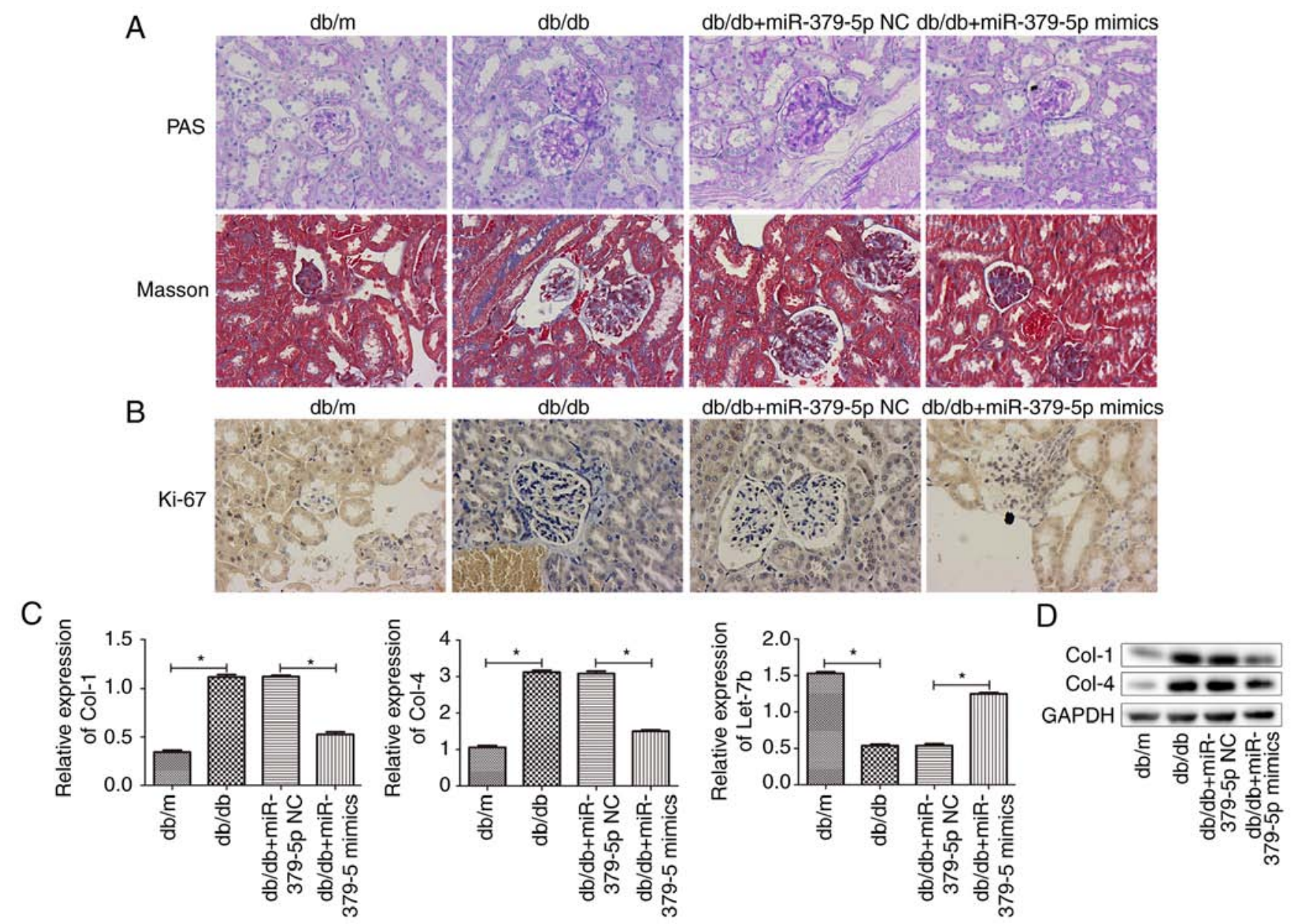

Figure 6. (A) PAS and Masson's trichrome staining of the glomeruli. (B) Ki-67 staining of the glomeruli. (C) RT-qPCR and (D) western blot analysis for Col4 and FN in kidney tissues. Data are expressed as the means \pm SEM. ${ }^{*} \mathrm{P}<0.05$ compared to $\mathrm{db} / \mathrm{m}$ mice or to $\mathrm{db} / \mathrm{db}$ mice.

upregulation of collagen expression in glomerular mesangial cells under diabetic conditions (14). Moreover, the LIN28/let-7 axis plays an important role in regulating glucose metabolism (47). Let-7a regulates glucose metabolism and insulin synthesis/secretion in type 2 diabetes mellitus by targeting the LIN28 pathway (48).

However, there are some limitations to this study. Mesangial cells have been used to study glomerular fibrosis in a number of studies $(49,50)$. The LIN28B/let-7 signaling pathway has been reported to be involved in cell energy metabolism in some cells. Further, we aim to examine the effects of miR-379-5p on podocytes in metabolism, not through fibrosis, particularly the influence of miR-379-5p in the process of podocyte diminution and disappearance in nephropathy in future studies.

In this study, another miRNA, miR-379-5p, was found to suppress renal fibrosis by regulating the LIN28B/let-7 axis in DN. miR-379-5p expression was downregulated and that of LIN28B was upregulated both in MMCs treated with HG and in glomeruli of $\mathrm{db} / \mathrm{db}$ mice. However, miR-379-5p mimics and agomir suppressed the expression of LIN28B in vitro and in vivo, respectively. The TargetScan database and luciferase reporter assay indicated that miR-379-5p directly bound to the 3' UTR of LIN28B and suppressed the expression of LIN28B. We verified that miR-379-5p regulated LIN28B in a HG-independent manner. In addition, let-7b, a target of LIN28B, was upregulated when the expression of miR-379-5p was increased. As the results of the overexpression of miR-379-5p, MMC proliferation and collagen protein accumulation were alleviated. It is thus suggested that miR-379-5p suppressed renal fibrosis by the regulating LIN28/let-7 axis in DN, which may lay the foundation for clinical treatment in the future.

In conclusion, the findings of this study suggested a coherent mechanism for the role of miR-379-5p in renal fibrosis during $\mathrm{DN}$, and provide new insight into the mechanisms of renal protection associated with miR-379-5p and LIN28/let-7, which may lead to a novel therapeutic strategy for DN.

\section{Acknowledgements}

Not applicable.

\section{Funding}

This study was supported by grants from the General Program of National Natural Science Foundation of China (grant no. 81774117); Postgraduate Research and Practice Innovation Program of Jiangsu Province (Grant no. KYCX18_1574) and National Youth Foundation of China (grant no. 81804027). 


\section{Availability of data and materials}

The datasets used during the current study are available from the corresponding author on reasonable request.

\section{Authors' contributions}

JYY designed research and revised the manuscript; NL and LJW performed the research analysis and wrote the manuscript; NL, LJW, WLX and SL analyzed the data. All authors have read and approved the final manuscript.

\section{Ethics approval and consent to participate}

All experiments were approved by the Animal Care and Use Committee of the Nanjing University of Chinese Medicine and complied with the Declaration of the National Institutes of Health Guide for the Care and Use of Laboratory Animals.

\section{Patient consent for publication}

Not applicable.

\section{Competing interests}

The authors declare that they have no competing interests.

\section{References}

1. Incidence and prevalence of ESRD. United States Renal Data System. Am J Kidney Dis 32 (2 Suppl 1): S38-S49, 1998.

2. Sun YM, Su Y, Li J and Wang LF: Recent advances in understanding the biochemical and molecular mechanism of diabetic nephropathy. Biochem Biophys Res Commun 433: 359-361, 2013.

3. Herbach N: Pathogenesis of diabetes mellitus and diabetic complications. Studies on diabetic mouse models. Pathologe 33 (Suppl 2): S318-S324, 2012 (In German).

4. Lagos-Quintana M, Rauhut R, Lendeckel W and Tuschl T: Identification of novel genes coding for small expressed RNAs. Science 294: 853-858, 2001.

5. Gholaminejad A, Abdul Tehrani H and Gholami Fesharaki M Identification of candidate microRNA biomarkers in diabetic nephropathy: A meta-analysis of profiling studies. J Nephrol 31: 813-831, 2018.

6. Allison SJ: Diabetic nephropathy: A lncRNA and miRNA megacluster in diabetic nephropathy. Nat Rev Nephrol 12: 713, 2016.

7. Alvarez ML and DiStefano JK: Towards microRNA-based therapeutics for diabetic nephropathy. Diabetologia 56: 444-456, 2013.

8. Cardenas-Gonzalez M, Srivastava A, Pavkovic M, Bijol V, Rennke HG, Stillman IE, Zhang X, Parikh S, Rovin BH, Afkarian $\mathrm{M}$, et al: Identification, confirmation, and replication of novel urinary MicroRNA biomarkers in lupus nephritis and diabetic nephropathy. Clin Chem 63: 1515-1526, 2017.

9. Liu F, Zhang ZP, Xin GD, Guo LH, Jiang Q and Wang ZX miR-192 prevents renal tubulointerstitial fibrosis in diabetic nephropathy by targeting Egr1. Eur Rev Med Pharmacol Sci 22: 4252-4260, 2018.

10. Peng J, Wu Y, Deng Z, Zhou Y, Song T, Yang Y, Zhang X, Xu T, Xia M, Cai A, et al: miR-377 promotes white adipose tissue inflammation and decreases insulin sensitivity in obesity via suppression of sirtuin-1 (SIRT1). Oncotarget 8: 70550-70563, 2017.

11. Wu J, Liu J, Ding Y, Zhu M, Lu K, Zhou J, Xie X, Xu Y, Shen X, Chen Y, et al: miR-455-3p suppresses renal fibrosis through repression of ROCK2 expression in diabetic nephropathy. Biochem Biophys Res Commun 503: 977-983, 2018.

12. Zhu X, Zhang C, Fan Q, Liu X, Yang G, Jiang Y and Wang L: Inhibiting microRNA-503 and microRNA-181d with losartan ameliorates diabetic nephropathy in KKAy mice. Med Sci Monit 22: 3902-3909, 2016.
13. Balzeau J, Menezes MR, Cao S and Hagan JP: The LIN28/let-7 pathway in cancer. Front Genet 8: 31, 2017.

14. Park JT, Kato M, Lanting L, Castro N, Nam BY, Wang M, Kang SW and Natarajan R: Repression of let-7 by transforming growth factor- $\beta 1$-induced Lin 28 up-regulates collagen expression in glomerular mesangial cells under diabetic conditions. Am J Physiol Renal Physiol 307: F1390-F1403, 2014.

15. Kim YS, Reddy MA, Lanting L, Adler SG and Natarajan R: Differential behavior of mesangial cells derived from 12/15-lipoxygenase knockout mice relative to control mice. Kidney Int 64: 1702-1714, 2003.

16. Livak KJ and Schmittgen TD: Analysis of relative gene expression data using real-time quantitative PCR and the 2(-Delta Delta C(T)) method. Methods 25: 402-408, 2001.

17. Shao Y, Lv C, Wu C, Zhou Y and Wang Q: Mir-217 promotes inflammation and fibrosis in high glucose cultured rat glomerular mesangial cells via Sirt1/HIF-1 $\alpha$ signaling pathway. Diabetes Metab Res Rev 32: 534-543, 2016.

18. Zhang Y, Wang S, Qian W, Ji D, Wang Q, Zhang Z, Wang S, Ji B, Fu Z and Sun Y: uc.338 targets $\mathrm{p} 21$ and cyclin D1 via PI3K/AKT pathway activation to promote cell proliferation in colorectal cancer. Oncol Rep 40: 1119-1128, 2018.

19. Kolset SO, Reinholt FP and Jenssen T: Diabetic nephropathy and extracellular matrix. J Histochem Cytochem 60: 976-986, 2012.

20. Zhou SX, Huo DM, He XY, Yu P, Xiao YH, Ou CL, Jiang RM, Li D and Li H: High glucose/lysophosphatidylcholine levels stimulate extracellular matrix deposition in diabetic nephropathy via plateletactivating factor receptor. Mol Med Rep 17: 2366-2372, 2018.

21. McWhorter ES, West RC, Russ JE, Ali A, Winger QA and Bouma GJ: LIN28B regulates androgen receptor in human trophoblast cells through Let-7c. Mol Reprod Dev: Jun 19, 2019 (Epub ahead of print). doi: 10.1002/mrd.23226 2019.

22. Kim CW, Vo MT, Kim HK, Lee HH, Yoon NA, Lee BJ, Min YJ, Joo WD, Cha HJ, Park JW and Cho WJ: Ectopic over-expression of tristetraprolin in human cancer cells promotes biogenesis of let-7 by down-regulation of Lin28. Nucleic Acids Res 40: 3856-3869, 2012.

23. Bera A, Das F, Ghosh-Choudhury N, Mariappan MM, Kasinath BS and Ghosh Choudhury G: Reciprocal regulation of miR-214 and PTEN by high glucose regulates renal glomerular mesangial and proximal tubular epithelial cell hypertrophy and matrix expansion. Am J Physiol Cell Physiol 313: C430-C447, 2017.

24. Chen D, Li Y, Mei Y, Geng W, Yang J, Hong Q, Feng Z, Cai G, Zhu $\mathrm{H}$, Shi S, et al: miR-34a regulates mesangial cell proliferation via the PDGFR- $\beta /$ Ras-MAPK signaling pathway. Cell Mol Life Sci 71: 4027-4042, 2014

25. Jee YH, Wang J, Yue S, Jennings M, Clokie SJ, Nilsson O, Lui JC and Baron J: Mir-374-5p, mir-379-5p, and mir-503-5p regulate proliferation and hypertrophic differentiation of growth plate chondrocytes in male rats. Endocrinology 159: 1469-1478, 2018.

26. Liang Y, Zhao G, Tang L, Zhang J, Li T and Liu Z: miR-100-3p and $\mathrm{miR}-877-3 \mathrm{p}$ regulate overproduction of IL-8 and IL-1 $\beta$ in mesangial cells activated by secretory $\operatorname{IgA}$ from IgA nephropathy patients. Exp Cell Res 347: 312-321, 2016.

27. Sun T, Yang J, Dong W, Wang R, Ma P, Kang P, Zhang H, Xie C, Du J and Zhao L: Down-regulated miR-15a mediates the epithelial-mesenchymal transition in renal tubular epithelial cells promoted by high glucose. Biosci Biotechnol Biochem 78: 1363-1370, 2014

28. Wang B, Yao K, Wise AF, Lau R, Shen HH, Tesch GH and Ricardo SD: miR-378 reduces mesangial hypertrophy and kidney tubular fibrosis via MAPK signalling. Clin Sci (Lond) 131: 411-423, 2017.

29. Wang X, Shen E, Wang Y, Jiang Z, Gui D, Cheng D, Chen T and Wang N: miR-196a regulates high glucose-induced mesangial cell hypertrophy by targeting p27kip1. J Lab Autom 20: 491-499, 2015.

30. Du B, Ma LM, Huang MB, Zhou H, Huang HL, Shao P, Chen YQ and Qu LH: High glucose down-regulates miR-29a to increase collagen IV production in HK-2 cells. FEBS Lett 584: 811-816, 2010 .

31. Liu H, Zhao J and Lv J: Inhibitory effects of miR-101 overexpression on cervical cancer SiHa cells. Eur J Gynaecol Oncol 38: 236-240, 2017.

32. Laddha SV, Nayak S, Paul D, Reddy R, Sharma C, Jha P, Hariharan M, Agrawal A, Chowdhury S, Sarkar C and Mukhopadhyay A: Genome-wide analysis reveals downregulation of miR-379/miR-656 cluster in human cancers. Biol Direct 8: $10,2013$. 
33. Khan S, Brougham CL, Ryan J, Sahrudin A, O'Neill G, Wall D, Curran C, Newell J, Kerin MJ and Dwyer RM: miR-379 regulates cyclin B1 expression and is decreased in breast cancer. PLoS One 8: e68753, 2013.

34. Nayak S, Aich M, Kumar A, Sengupta S, Bajad P, Dhapola P, Paul D, Narta K, Purkrait S, Mehani B, et al: Novel internal regulators and candidate miRNAs within miR-379/miR-656 miRNA cluster can alter cellular phenotype of human glioblastoma. Sci Rep 8: 7673, 2018.

35. Huang DJ, Huang JZ, Yang J, Li YH, Luo YC, He HY and Huang HJ: Bioinformatic identification of IGF1 as a hub gene in hepatocellular carcinoma (HCC) and in-vitro analysis of the chemosensitizing effect of miR-379 via suppressing the IGF1/IGF1R signaling pathway. Eur Rev Med Pharmacol Sci 20: 5098-5106, 2016.

36. Hao GJ, Hao HJ, Ding YH, Wen H, Li XF, Wang QR and Zhang BB: Suppression of EIF4G2 by miR-379 potentiates the cisplatin chemosensitivity in nonsmall cell lung cancer cells. FEBS Lett 591: 636-645, 2017.

37. Yi J and An Y: Circulating miR-379 as a potential novel biomarker for diagnosis of acute myocardial infarction. Eur Rev Med Pharmacol Sci 22: 540-546, 2018.

38. Xu Z, Han Y, Liu J, Jiang F, Hu H, Wang Y, Liu Q, Gong Y and Li X: miR-135b-5p and miR-499a-3p promote cell proliferation and migration in atherosclerosis by directly targeting MEF2C. Sci Rep 5: 12276, 2015.

39. Cheng SW, Tsai HW, Lin YJ, Cheng PN, Chang YC, Yen CJ, Huang HP, Chuang YP, Chang TT, Lee CT, et al: Lin28B is an oncofetal circulating cancer stem cell-like marker associated with recurrence of hepatocellular carcinoma. PLoS One 8: e80053, 2013.

40. King CE, Cuatrecasas M, Castells A, Sepulveda AR, Lee JS and Rustgi AK: LIN28B promotes colon cancer progression and metastasis. Cancer Res 71: 4260-4268, 2011.

41. Alajez NM, Shi W, Wong D, Lenarduzzi M, Waldron J, Weinreb I and Liu FF: Lin28b promotes head and neck cancer progression via modulation of the insulin-like growth factor survival pathway. Oncotarget 3: 1641-1652, 2012.

42. Kugel S, Sebastian C, Fitamant J, Ross KN, Saha SK, Jain E, Gladden A, Arora KS, Kato Y, Rivera MN, et al: SIRT6 suppresses pancreatic cancer through control of lin28b. Cell 165: 1401-1415, 2016.
43. Piskounova E, Polytarchou C, Thornton JE, LaPierre RJ, Pothoulakis C, Hagan JP, Iliopoulos D and Gregory RI: Lin28A and Lin28B inhibit let-7 microRNA biogenesis by distinct mechanisms. Cell 147: 1066-1079, 2011.

44. McDaniel K, Huang L, Sato K, Wu N, Annable T, Zhou T, Ramos-Lorenzo S, Wan Y, Huang Q, Francis H, et al: The let-7/Lin28 axis regulates activation of hepatic stellate cells in alcoholic liver injury. J Biol Chem 292: 11336-11347, 2017.

45. Liang H, Liu S, Chen Y, Bai X, Liu L, Dong Y, Hu M, Su X, Chen Y,Huangfu L, et al: miR-26a suppresses EMT by disrupting the Lin28B/let-7d axis: Potential cross-talks among miRNAs in IPF. J Mol Med (Berl) 94: 655-665, 2016.

46. Hills CE and Squires PE: TGF-betal-induced epithelial-to-mesenchymal transition and therapeutic intervention in diabetic nephropathy. Am J Nephrol 31: 68-74, 2010.

47. Zhu H,Shyh-Chang N, Segre AV, Shinoda G, Shah SP, Einhorn WS, Takeuchi A, Engreitz JM, Hagan JP, Kharas MG, et al: The Lin28/let-7 axis regulates glucose metabolism. Cell 147: 81-94, 2011.

48. Frost RJ and Olson EN: Control of glucose homeostasis and insulin sensitivity by the Let-7 family of microRNAs. Proc Nat Acad Sci USA 108: 21075-21080, 2011.

49. Ji J, Tao P and He L: Kangxianling decoction prevents renal fibrosis in rats with 5/6 nephrectomy and inhibits Ang II-induced ECM production in glomerular mesangial cells. J Pharmacol Sci 139: 367-372, 2019.

50. Lee EJ, Kang MK, Kim DY, Kim YH, Oh H and Kang YH: Chrysin inhibits advanced glycation end products-induced kidney fibrosis in renal mesangial cells and diabetic kidneys. Nutrients 10: E882, 2018

This work is licensed under a Creative Commons Attribution-NonCommercial-NoDerivatives 4.0 International (CC BY-NC-ND 4.0) License. 